\title{
Climatic variation affects clutch phenology in Agassiz's desert tortoise Gopherus agassizii
}

\author{
Jeff Lovich ${ }^{1, *}$, Mickey Agha ${ }^{1}$, Meaghan Meulblok ${ }^{1}$, Kathie Meyer $^{2}$, Josh Ennen $^{1,4}$, \\ Caleb Loughran ${ }^{1,5}$, Sheila Madrak ${ }^{1,6}$, Curtis Bjurlin ${ }^{3}$ \\ ${ }^{1}$ U.S. Geological Survey, Southwest Biological Science Center, Flagstaff, Arizona 86011, USA \\ ${ }^{2}$ U.S. Forest Service, Front Country Ranger District, San Bernardino National Forest, Lytle Creek, California 92358, USA \\ ${ }^{3}$ Stantec Consulting Services, Inc., Cottage Grove, Wisconsin, 53527, USA \\ ${ }^{4}$ Present address: TN-SCORE, University of Tennessee, Knoxville, Tennessee 37996, USA \\ ${ }^{5}$ Present address: Central Washington University, Department of Biological Sciences, Ellensburg, Washington 98926, USA \\ ${ }^{6}$ Present address: San Diego State University/UC Davis, Department of Biology, San Diego, California 92182, USA
}

\begin{abstract}
There is concern about how climate change might affect Agassiz's desert tortoise Gopherus agassizii, a threatened species. We studied the effects of climatic variation on clutch phenology of a population at a wind energy generation facility for 7 field seasons between 1997 and 2011. Using X-radiography, we quantified the following phenophases based on the number of calendar days to each event from 1 January: appearance and disappearance of 65 first and 53 second clutches, and inter-clutch intervals between first and second clutches. Although third clutches were rare $(n=8)$, they were observed in 5 of $7 \mathrm{yr}$ and were produced by 8 different females, all of which produced a third clutch only once during the study. Shelled eggs were visible from as early as 11 April to as late as 28 July, and the overall time span that eggs were visible differed among years. After controlling for maternal effects, we observed statistically significant inter-annual variation in all phenophases except for inter-clutch interval. Clutch appearance was late in cool years relative to warm years and especially late after 2 consecutive cool years. Using degree day (DD) methodology, we calculated DD accumulation during the post-hibernation and nesting season for each year. We then used DD estimates for mean date of first clutch appearance to predict the mean date of first clutch appearance in subsequent years with 1 to $10 \mathrm{~d}$ accuracy for all but $1 \mathrm{yr}$ (1998) with El Niño conditions. Clutch phenology appears to be correlated with inter-annual variation in climate and may be influenced by climatic events in previous years.
\end{abstract}

KEY WORDS: Climate $\cdot$ Desert tortoise $\cdot$ Clutch phenology $\cdot$ Degree days $\cdot$ Biofix Resale or republication not permitted without written consent of the publisher

\section{INTRODUCTION}

Climate, especially temperature and precipitation, exerts a strong influence on the life cycles of most organisms. This is especially true for ectotherms like desert tortoises that need to self-regulate their body temperature behaviorally and in response to environmental conditions (Zimmerman et al. 1994). As a result, they and other ectotherms, are particularly sensitive to climatic variation (Barrows 2011) due possibly to fitness consequences imposed by varying environmental temperatures (Huey \& Berrigan 2001). Phenology is the study of the timing and environmental causes, both biotic and abiotic, of recurring biological events in life cycles (Mitchell 1979, Stenseth \& Mysterud 2002), and perhaps the simplest indicator of ecological responses to climate change (Walther et al. 2002). For oviparous organisms, clutch phenology includes critical events in their life histories such as the rate of egg development, egg retention time (Buhlmann et al. 1995), timing of oviposition, inter-clutch intervals, incubation duration, and 
time of hatching. This continuum corresponds to the pre-paritive developmental period defined by Morafka et al. (2000).

For these reasons, clutch phenology has a potentially significant effect on nest survival and success, incubation time, the ability to produce multiple clutches (Wallis et al. 1999), and even hatchling sex ratio (Lewis-Winokur \& Winokur 1995, Baxter et al. 2008) in species with environmental sex determination (Ewert \& Nelson 1991, Mitchell \& Janzen 2010). In addition, clutch phenology in organisms with environmental sex determination can affect maternal and hatchling fitness via its proximate role in determining exposure to post-oviposition environmental (incubation) temperatures that differentially affect the fitness of male and female offspring (Shine 1999). The relationship between clutch phenology and post-ovipositional environmental temperatures has the potential to influence developmental rate, phenotype, post-hatching growth rate, and post-emergence survival of hatchlings (Lewis-Winokur \& Winokur 1995, Rhen \& Lang 1995, 1999, Freedberg et al. 2001, Ashmore \& Janzen 2003, Schwanz et al. 2010). However, others report that nesting phenology exhibits a low potential for natural selection to influence this maternal factor in turtles (Warner et al. 2010).

The timing of egg production and oviposition may be particularly important to animals living in highly variable environments (Gibbons \& Nelson 1978). Agassiz's desert tortoise Gopherus agassizii, a federally threatened species, is a good model in this regard due to the extreme temporal and spatial variability of food resources available to both reproductive adults (Peterson 1996, Henen 2002a,b) and their progeny (Morafka 1994, Morafka et al. 2000). Much of the variability in food resources is due to annual and spatial variation in the amount and timing of rainfall (Beatley 1974, Bowers 2005) that stimulates germination of food plants in the Mojave and Sonoran Deserts where G. agassizii lives. Clutch phenology must be properly timed to allow emerging hatchlings to take advantage of resource availability in such an unpredictable environment (Rostal et al. 1994). The fitness consequences are significant: if rainfall is not adequate to produce food plants for the herbivorous tortoise, it may have catastrophic effects on population recruitment and survival, leading to local extirpations (Morafka 1994) and ultimately confounding efforts to recover this declining species (US Fish and Wildlife Service 2011). Rainfall is thus an important exogenous factor that influences the reproductive ecology of $G$. agassizii through its direct effect on the germination of food plants and atten- dant effects on clutch frequency and the percentage of adult females that reproduce in a given year (Turner et al. 1986, Lovich et al. 1999).

However, the role of temperature as a dominant factor in seasonal reproductive cyclicity (Licht 1983) cannot be overlooked due to its influence on physiology and the rate of egg development (Rostal et al. 1994) and incubation time (Lewis-Winokur \& Winokur 1995) in Gopherus agassizii. Rostal et al. (1994, p. 79) observed that vitellogenesis and ovarian maturation in G. agassizii coincided with peak air temperatures and concluded that 'air temperature appears to be a relatively constant environmental cue which coincides with physiological changes in reproduction.' Nagy \& Medica (1986) concluded that the physiology and ecology of $G$. agassizii is greatly influenced by both temperature and rain. In the closely related species, G. flavomarginatus, peak air temperatures correspond with elevated estradiol levels (Gonzalez-Trapaga et al. 2000), further confirming the importance of temperature to the reproductive cycle of tortoises.

We examined clutch phenology in a population of Gopherus agassizii near Palm Springs, California, during 7 field seasons spanning 15 yr. Local climate varied substantially during the study, including both drought and El Niño conditions (Ennen et al. 2012a), and years characterized by 'warm' versus 'cool' spring temperatures. Wide variation in clutch phenology attended climatic fluctuations, and we wanted to examine their interrelationships to better understand the possible effects of climate change on the reproductive ecology of this threatened species. Thus, our study had 2 objectives. First, we wanted to quantitatively measure variation in clutch phenology among years and determine how it was correlated with inter-annual climatic variation as measured by temperature. Second, we aimed at developing a model to predict the mean timing of first clutch appearance in $\mathrm{X}$-radiographs relative to degree day (DD) accumulation in a given year. We describe a method that successfully predicts mean date of first clutch appearance with an accuracy of 1 to $10 \mathrm{~d}$ for all but 1 yr with El Niño conditions.

\section{MATERIALS AND METHODS}

\section{Study site description}

We studied the reproductive ecology of a population of Gopherus agassizii from 1997 to 2011 during 7 field seasons (1997, 1998, 1999, 2000, 2009, 2010, and 2011). Partial details of those studies are provided by 
Lovich et al. (1999) and Ennen et al. (2012b), and a more detailed review of reproductive output examining clutch and egg size is forthcoming ( $\mathrm{J}$. Lovich unpubl. data). The study site, known locally as the Mesa wind farm, is located at the extreme western end of the Sonoran Desert ecosystem (Burk 1977, Lovich et al. 2011b) in the foothills of the San Bernardino Mountains near Palm Springs, California, USA. A large wind energy generation facility has operated at the site since shortly after 1983 and currently includes 460 turbines and the associated infrastructure. Elevations occupied by G. agassizii in the area ranged from 600 to $900 \mathrm{~m}$. Habitat at the study site is characterized by creosote bush Larrea tridentata scrub, but plants representative of coastal sage scrub communities (California sagebrush Artemisia californica), chaparral communities (chamise Adenostema fasciculatum), and the nearby Mojave Desert (spiny hopsage Grayia spinosa) are also present due to the semi-desert chaparral nature of the area. Much of the study site was burned by a large wildland fire in 1995, with little apparent effect on the home ranges, body condition indices, and annual reproductive output of female G. agassizii (Lovich et al. 2011a). Long-term winter (October to March) mean precipitation is $114 \mathrm{~mm}$, most of which falls as rain (Ennen et al. 2012a). Additional details of the study site and tortoise population are given by Lovich \& Daniels (2000) and Lovich et al. (2011b).

\section{Field techniques}

As described by Lovich et al. (1999, 2011a,b), each tortoise received a unique identification code based on notching combinations of marginal scutes with a triangular file. In addition, correspondingly numbered small paper tags were affixed to the posterior carapace with clear epoxy. Radio transmitters were attached (Boarman et al. 1998) to female tortoises ( 7 to 17 females were radio-tracked per year), and they were tracked from April (late March in 1998, 1999, 2000, and 2010) to the end of July, when shelled eggs were normally visible via X-radiography. Females were measured once at the beginning of each field season using straight line carapace length and weighed at every capture. Each individual was Xradiographed (Hinton et al. 1997) in the field at 7 to $10 \mathrm{~d}$ intervals when captured from April (or March) through July. X-radiographs were taken to assess clutch size and frequency using film-based radiography and rare earth cassettes from 1997 to 2010, and digital radiography equipment in 2011.
To assess the correspondence between the date when a clutch was last seen in an X-radiograph and when the female oviposited, we located nests at Mesa in 2011 using thread-trailing. Once heavily shelled eggs in first and second clutches were detected in X-radiographs, thread-trailing devices (Claussen et al. 1997) were attached to the posterior end of a female's carapace. We located each female with radiotelemetry on a daily basis and weighed her. If significant weight loss occurred that approximated her known clutch size multiplied by the average weight of each egg $(\sim 30 \mathrm{~g})$, then oviposition was demonstrated. We then retraced the trail of thread and searched for the nest, concentrating on burrows occupied during the preceding days. Actual date of oviposition was thus based on either detection of eggs in the ground or inferred from significant weight loss (Turner et al. 1984, 1986). After oviposition, the thread-trailing device was removed until a second clutch was detected on a subsequent $\mathrm{X}$-radiograph.

\section{Clutch phenology}

We counted calendar days (Wilimovsky 1990) from January 1 (inclusive) to various clutch phenological events, accounting for the leap year in 2000. Phenophases measured included the date a first clutch appeared in a female (C1APPEAR), the date the first clutch disappeared in a female (C1DISAPPEAR), the date a second clutch appeared in a female (C2APPEAR), and the date a second clutch disappeared in a female (C2DISAPPEAR). These variables corresponded to the onset and termination attributes of phenophases recommended by Mitchell (1979). Clutch intervals (number of days a clutch was visible in X-radiographs) were not included in our calculations if the tortoise was radioed late in the reproductive season (mid-April or later) because there was no way to determine clutch order (first, second, or third), as was the case with 2 females in 1997, 1 in 1998 and 1 in 2000. Data for the second clutch of 1 female in 1998 were discarded because she died before eggs were dropped. In other tortoises we observed the beginning of clutches but not the end, so we included only the former dates in our analyses. The sample size for third clutches was small $(\mathrm{n}=8)$; therefore, they are not included in statistical analyses unless indicated. Inter-clutch intervals were measured as number of days between when the first clutch was last visible and when the second clutch was first visible. 


\section{Climate data and DD calculations}

Ambient air temperature data were collected from 1 March to 31 July in all years, corresponding to the period from when tortoises typically emerge from hibernation to when nesting ends at the study site (J. Lovich unpubl. data). While the authors recognize that body temperatures or tortoise burrow temperatures would have been preferable to ambient air temperatures, those data were not available. Hourly air temperatures were collected using $\mathrm{HOBO}{ }^{\circledR}$ temperature data loggers deployed at the study site from 1997 to 2000. During the period from 2009 to 2011, we collected air temperatures from local remote automated weather stations (RAWS) using the MesoWest website (http://mesowest.utah.edu/index. $\mathrm{html}$ ) and data listed for the white water station (Station ID WWAC1), also located at our study site. In the case of 2 mo of missing temperature data, one in 1997 and one in 2000, a linear regression equation was created relating known study site air temperatures to time-matched air temperatures at 2 nearby RAWSs: (1) Palm Springs, California (Station ID KPSP), and (2) Mill Creek, California (Station ID MMKC1). Incomplete RAWS temporal coverage necessitated the use of 2 recording stations. Both regressions were significant at $\mathrm{p}<0.001$ and yielded high $\mathrm{R}^{2}$ values (0.729 and 0.864 , respectively). We then used the resulting regression equations to replace missing data with estimates of air temperature based on RAWS data.

A DD model which quantifies the daily regional climate via 'heat' unit (e.g. DD) accumulation was adapted to predict phenological stages of clutch development in Gopherus agassizii. The accumulation of DDs is commonly used by agriculturists to monitor development of biological processes (Baskerville \& Emin 1969) related to crop production (Snyder 1985). In addition, DD accumulation has been used in other phenological studies of turtle reproductive ecology to estimate the date of first nesting (Obbard \& Brooks 1987), sex determination (Valenzuela 2001), and nesting frequency (Iverson 1991). A simplified DD calculation was provided by Snyder (1985) that requires knowledge of minimum and maximum daily temperatures, a developmental or related temporal point of reference called a biofix, and a threshold temperature. A biofix is defined as a biological event, or indicator of a developmental event, that is used as a starting point for DD calculations (Chhagen \& Stevens 2007).

For our calculations we selected the typical termination of hibernation as a biofix for Gopherus agas- sizii. In response to yearly variation in environmental temperatures, G. agassizii typically terminates hibernation in early March (Woodbury \& Hardy 1948, Luckenbach 1982, Rautenstrauch et al. 1998). Because of this timing, we set the biofix date at 1 March, though we recognize that the actual date of emergence from hibernation varies throughout the range of G. agassizii (Nussear et al. 2007, Ernst \& Lovich 2009). Beginning on 1 March, DD accumulation was calculated until the end of the nesting season (set at 31 July; J. Lovich unpubl. data), using the method of Snyder (1985). To determine the threshold temperature associated with our biofix, we used air temperatures for both October and March, as these are the months when G. agassizii typically enters and terminates hibernation, respectively (Rautenstrauch et al. 1998, Ernst \& Lovich 2009). Following Nussear et al. (2007) we averaged hourly temperatures per day during March and October in all study years (1997 to 2000, 2009 to 2011) to estimate a threshold temperature of $17.77^{\circ} \mathrm{C}$ (decimal places for calculation only recognizing accuracies of +1 or -1 degree) for entering and leaving hibernation at Mesa. Our estimated threshold temperature is between the values of 15 and $20^{\circ} \mathrm{C}$ reported by Woodbury \& Hardy (1948) and Voigt (1972), respectively, for termination of hibernation and initiation of activity by G. agassizii. When the daily minimum air temperature rose above our threshold temperature of $17.77^{\circ} \mathrm{C}$, DD accumulation was calculated with the following equation:

$$
\begin{gathered}
\mathrm{DD}=(\text { max. air temp. + min. air temp. }) / \\
2-\text { threshold temp. }
\end{gathered}
$$

When the daily minimum air temperature was below the threshold temperature of $17.77^{\circ} \mathrm{C}$, DD was calculated using the following equations:

$$
\begin{gathered}
W=\text { (max. air temp. }- \text { min. air temp.) } / 2 \\
R=\text { (threshold temp. }- \text { min. air temp.) } / \\
\text { (max. air temp. }- \text { min. air temp.) }
\end{gathered}
$$

DDs were then calculated as: $W \times N$ where $N$ is a fraction of $W$ corresponding to the relative position ratio of $R$. Table 1 in Snyder (1985) displays $R$ values and corresponding $N$ values that are ratios and fractions of a sine curve temperature approximation. If the threshold temperature exceeds the maximum air temperature, then $N=0$. DD values were then totaled to the date of a selected clutch phenophase to produce DD accumulation.

We used DD accumulation values to predict nesting dates in subsequent years based on 2 widely separated time periods with distinctly different 


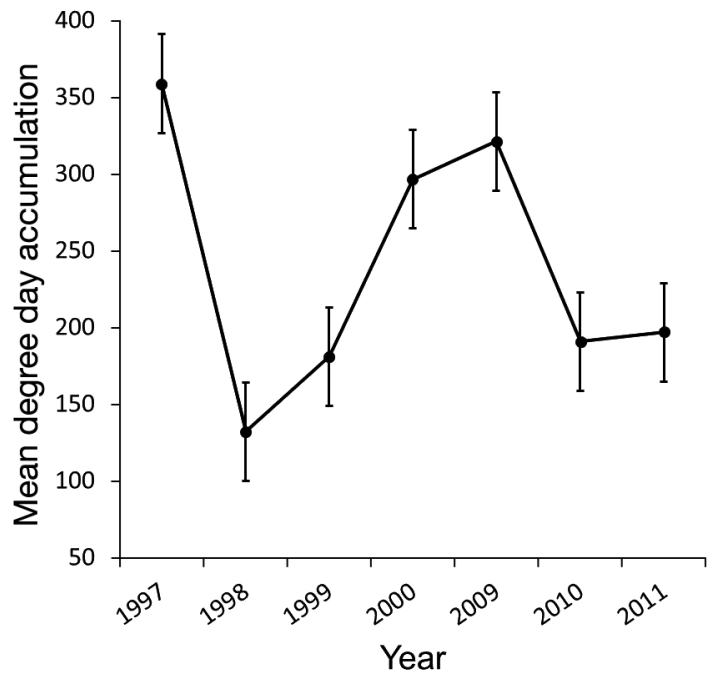

Fig. 1. Mean degree day (DD) accumulation values and associated standard errors for all years of study. Line connecting means is for reference only. Means are based on the sum of daily DD accumulation values from 1 March to 31 July divided by the number of days in that time period; this corresponds to the period from emergence from hibernation through the end of the egg-laying season for Gopherus agassizii near Palm Springs, California, USA

climates (Fig. 1). For the first time period, we used the DD accumulation value for the mean date of C1APPEAR in 1997 (172 DD) to predict the dates for mean C1APPEAR in 1998, 1999, and 2000. Based on this analysis, our expectation was that mean C1APPEAR in 1998, 1999, and 2000 would occur on dates when the DD accumulation value for 1997 was achieved or exceeded for the first time. For the second time period, we used DD accumulation values coincident with mean C1APPEAR from our biofix in 2009 (120 DD) to predict mean C1APPEAR in 2010 and 2011, again with the assumption that mean C1APPEAR in subsequent years would occur when DD accumulation values were greater than or equal to the value calculated for 2009. The mean number of calendar days to C1APPEAR for all tortoises was used rather than the actual date of C1APPEAR for each individual tortoise because we could not confirm the absolute first appearance and disappearance of shelled eggs due to our 7 to $10 \mathrm{~d}$ X-radiograph intervals.

Mean DD accumulation values among study years, for the period covering emergence from hibernation through nesting season period are shown in Fig. 1. These values reflect a measure of overall temperature accumulation for the time period. Higher mean DD values reflect 'warmer' years relative to 'cooler' years with lower means.

\section{Statistical analyses}

We used hierarchical linear mixed models to test for differences among years in the mean number of calendar days from 1 January to the beginning or end of our phenophases (C1APPEAR, C1DISAPPEAR, C2APPEAR, C2DISAPPEAR, and inter-clutch intervals) after controlling for maternal effects (since many of the same females were X-radiographed from year to year). Year was treated as a fixed effect. To control for maternal effects individual tortoises were treated as a random effect in the covariance structure. Tukey's Honestly Significant Difference test was used for all pairwise comparisons among mean phenophases for each year. We expected that the 5 phenological events would exhibit similar trends due to their serial interconnectedness within years, but tested them all due to wide temporal overlap between first, second, and third clutches both within and among years. Due to a protocol change in 2000, we had very few C2DISAPPEAR observations since tortoises were rarely X-radiographed after detection of a second clutch. We used the same mixed model analysis to test for differences in the mean number of days that first and second clutches were visible across years after controlling for maternal effects. Statistical tests were conducted using SYSTAT 13 software with an alpha level of 0.05. Data were tested for normality and transformed as needed.

\section{RESULTS}

Mean square root-transformed DD accumulation values differed dramatically among years $(F=19.47$; $\mathrm{df}=6,1064 ; \mathrm{p}<0.001$ ) as shown in Fig. 1 , reflecting substantial climatic variation. The earliest date that first clutches $(\mathrm{n}=65)$ were visible was 11 April (Fig. 2) for 2 tortoises (1997). The latest date that first clutches disappeared was 13 July in 1999, and only 3 first clutches extended into July (1999, 2000, 2011). The earliest date that second clutches $(\mathrm{n}=53)$ were visible was 1 May (1997). The latest date that second clutches were visible was 20 July for 3 tortoises (1999). The earliest date that third clutches were visible was 16 June (2009). The latest date that third clutches were visible was 28 July; that female was not X-radiographed again that year so it is possible that she oviposited in early August.

Based on beginning and end dates, eggs were visible for intervals of the following numbers of days in each year (Fig. 2): 84 (1997), 105 (1998), 71 (1999), 81 (2000), 58 (2009), 92 (2010), and 89 (2011). A Chi- 


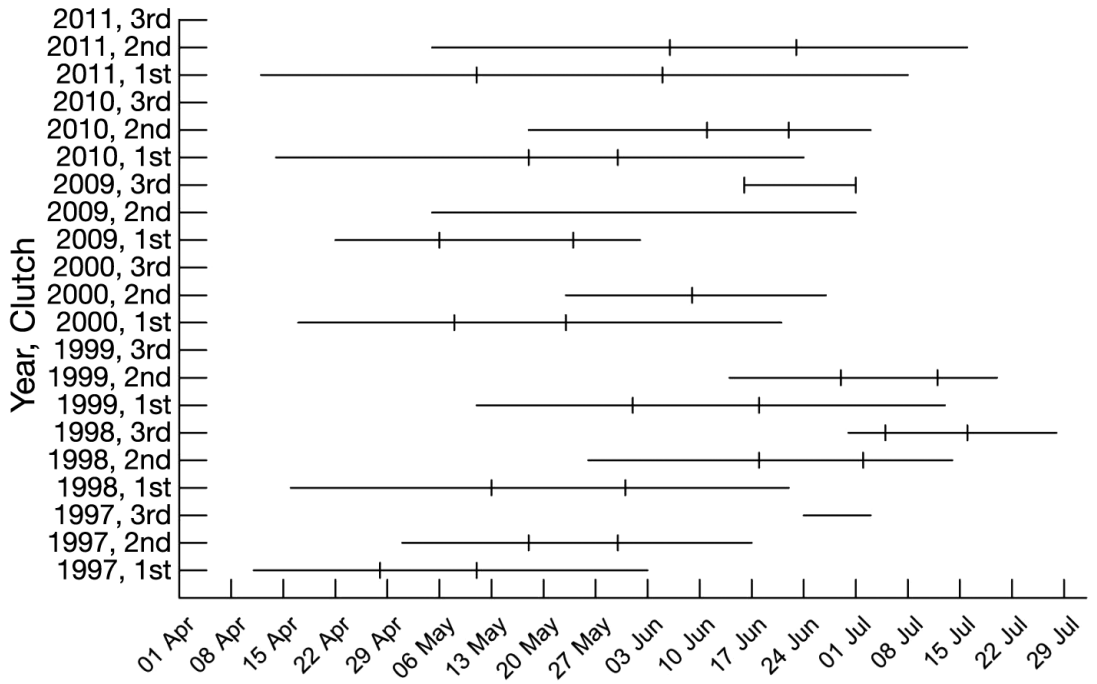

Fig. 2. Gopherus agassizii. Clutch phenology of desert tortoises determined by $\mathrm{X}$-radiography. Horizontal lines represent range of dates when shelled eggs were visible in X-radiographs for the respective year and clutch number. Vertical tick marks show mean clutch appearance and disappearance dates for all clutches of that order (first, second, or third) in a particular year. Lines with no tick marks represent single clutches. The line for second clutches in 2009 does not have a tick mark since the means for appearance and disappearance were essentially the same (4 June)

squared test revealed that these intervals were significantly different from the expected proportionally equal values $\left(\chi^{2}=16.59, \mathrm{df}=6, \mathrm{p}=0.01\right)$. Summary statistics for the number of days that individual first, second, and third clutches were visible in all years, as well as inter-clutch intervals between first and second clutches, are shown in Table 1.

There was no difference in the mean number of days that first and second clutches were visible after controlling for maternal effects in a mixed-model analysis of variance (ANOVA) $(F=2.189$; $\mathrm{df}=1,95$; $\mathrm{p}=0.142$ ), and that relationship was consistent across years $(F=0.004 ; \mathrm{df}=1,95 ; \mathrm{p}=0.953)$. Third clutches were rare $(n=8)$ and were observed in 5 out of 7 field seasons during our $15 \mathrm{yr}$ study. These third clutches were produced by 8 different females, all of which produced a third clutch only once during the entire

Table 1. Gopherus agassizii. Summary statistics for the number of days that the first, second and third clutches were visible in X-radiographs, and the associated inter-clutch intervals

\begin{tabular}{|lccc|}
\hline & Mean (d) & SD & Range (d) \\
\hline 1st clutch & 17.4 & 6.5 & $7-35$ \\
2nd clutch & 15.5 & 4.6 & $8-29$ \\
3rd clutch & 10.3 & 3.3 & $8-15$ \\
Days between 1st and 2nd & 16.6 & 2.8 & $7-34$ \\
Days between 2nd and 3rd & 28.3 & 10.1 & $7-39$ \\
\hline
\end{tabular}

study period. If we disregard 4 third clutches with incomplete data (beginning or end dates unknown), an ANOVA comparing the mean number of days that first, second, and third clutches were visible (Table 1) demonstrated a statistically significant $(F=$ $3.474 ; \mathrm{df}=2,100 ; \mathrm{p}=0.035$ ) difference. Mean number of days clutches were visible appeared to be different between first and third clutches, and the highly conservative (especially for unequal sample sizes like ours) Tukey's test for pairwise comparisons detected an almost significant difference between those 2 means $(p=0.052)$. The beginning of first clutches (C1APPEAR) was highly correlated with the beginning of second clutches (C2APPEAR) in the same tortoise, as shown by a Pearson's correlation coefficient of $0.88(\mathrm{p}<0.001)$.

Actual dates of oviposition for both first and second clutches in 2011 (based on finding nests and inferring oviposition dates based on significant weight loss) ranged from 1 to $12 \mathrm{~d}$ after eggs were last detected in X-radiographs, with a mean of $6.4 \mathrm{~d}$ and SD of $2.8(\mathrm{n}=10 \mathrm{fe}-$ males and 14 clutches). Precision increased based on detection of actual nests (as opposed to including inferred oviposition dates), with intervals ranging from 1 to $8 \mathrm{~d}$, with a mean of 5.8 ( $\mathrm{n}=8$ females and 8 clutches). Using only inferred oviposition dates (based on weight loss), intervals increased ranging from 5 to $12 \mathrm{~d}$, with a mean of 7.3 ( $\mathrm{n}=6$ females and 6 clutches).

Several clutch phenophases were significantly correlated with climate, measured by mean DD accumulation values from 1 March to 31 July of each year (Fig. 1), as shown by the following Pearson correlation coefficients and their associated probabilities: mean C1DISAPPEAR days since 1 January, $r=-0.76$, $p=0.049$; mean C2APPEAR days since 1 January, $r=$ $-0.78, p=0.039$; mean C2DISAPPEAR days since 1 January, $r=-0.79, p=0.035$; and mean number of days first clutches were visible, $r=-0.78, p=0.038$. As expected, clutch phenology was significantly delayed in cooler years relative to warmer years. Two phenophases were not significantly correlated with DD accumulation values from 1 March to 31 July, although one was almost so: mean C1APPEAR days since 1 January, $r=-0.717, p=0.070$ and mean number of days second clutches were visible, $r=0.06, p=$ 0.900 . 
Mixed-model ANOVAs identified a significant difference among years in the means for C1APPEAR, C1DISAPPEAR, C2APPEAR, and C2DISAPPEAR after controlling for maternal effects (Table 2). Differences among years are shown in Fig. 3 for both first and second clutches. Pairwise comparisons among years demonstrated that 1999 had very different clutch phenology from most other years (Table 3); that year was the latest for C1APPEAR (11 May) and the only year with no visible clutches in April. Interclutch intervals between first and second clutches did not differ significantly among years $(F$ ratio $=$ 0.645 ; df $=6,28 ; \mathrm{p}=0.694$ ).

Based on a DD accumulation of 172 DD in 1997 (Fig. 4) at the mean date for C1APPEAR, we predicted that mean C1APPEAR in 1998 would occur when accumulated DDs for that year were greater

Table 2. Gopherus agassizii. Results from hierarchical linear mixed-models testing for differences (see 'Materials and methods: Statistical analyses') among years in the mean number of days from 1 January to phenological events related to clutch production after controlling for maternal effects: days to appearance of first clutch (C1APPEAR), days to disappearance of first clutch (C1DISAPPEAR), days to appearance of second clutch (C2APPEAR), and days to disappearance of second clutch (C2DISAPPEAR). Refer to 'Results' for further details

\begin{tabular}{|lccc|}
\hline Event & F-ratio & $\mathrm{df}$ & $\mathrm{p}$ \\
\hline C1APPEAR & 8.53 & 6,40 & $<0.001$ \\
C1DISAPPEAR & 13.07 & 6,34 & $<0.001$ \\
C2APPEAR & 8.37 & 6,27 & $<0.001$ \\
C2DISAPPEAR & 14.95 & 6,12 & $<0.001$ \\
\hline
\end{tabular}

than or equal to the same value; this occurred on 25 June. The actual date for mean C1APPEAR in the El Niño year of 1998 was 13 May (80 DD), varying appreciably from our prediction by $43 \mathrm{~d}$. Using the same technique and threshold value from 1997 (172 DD), we tested the model's ability to predict mean C1APPEAR in 1999 and 2000. For 1999, DD accumulation first equaled 172 DD on 11 June, but mean C1APPEAR occurred on 1 June at 153 DD, $10 \mathrm{~d}$ earlier than our prediction. For 2000, DD accumulation equaled 172 DD on 8 May, and mean C1APPEAR that year occurred on 8 May at the same value and on the same day of our prediction.

Mean C1APPEAR in 2009 occurred on 5 May, when DD accumulation was equal to 120 DD. Based on this value in 2009, we predicted that mean C1APPEAR would occur on 19 May 2010, since that was the first time DD accumulation was greater than or equal to $120 \mathrm{DD}$. The actual date for mean C1APPEAR in 2010 was 18 May, when DD accumulation was $116 \mathrm{DD}$, only $1 \mathrm{~d}$ off from our prediction. For 2011, we predicted that mean C1APPEAR would occur on 6 May. The actual date for mean C1APPEAR in 2011 was 9 May, when DD accumulation was 123 DD, 3 d later than predicted. Our predicted dates for all years thus had an accuracy of 1 to $43 \mathrm{~d}$ relative to the estimates. Removing 1998 as anomalous due to the influence of El Niño weather conditions at Mesa (Ennen et al. 2012a) improved our range of estimates to 1-10 d accuracy. Of the 9 individual females that produced eggs in 1997, 6 (66.6\%) oviposited later in the season in 1998 than they did in 1997, further reflecting the anomalous conditions of 1998.
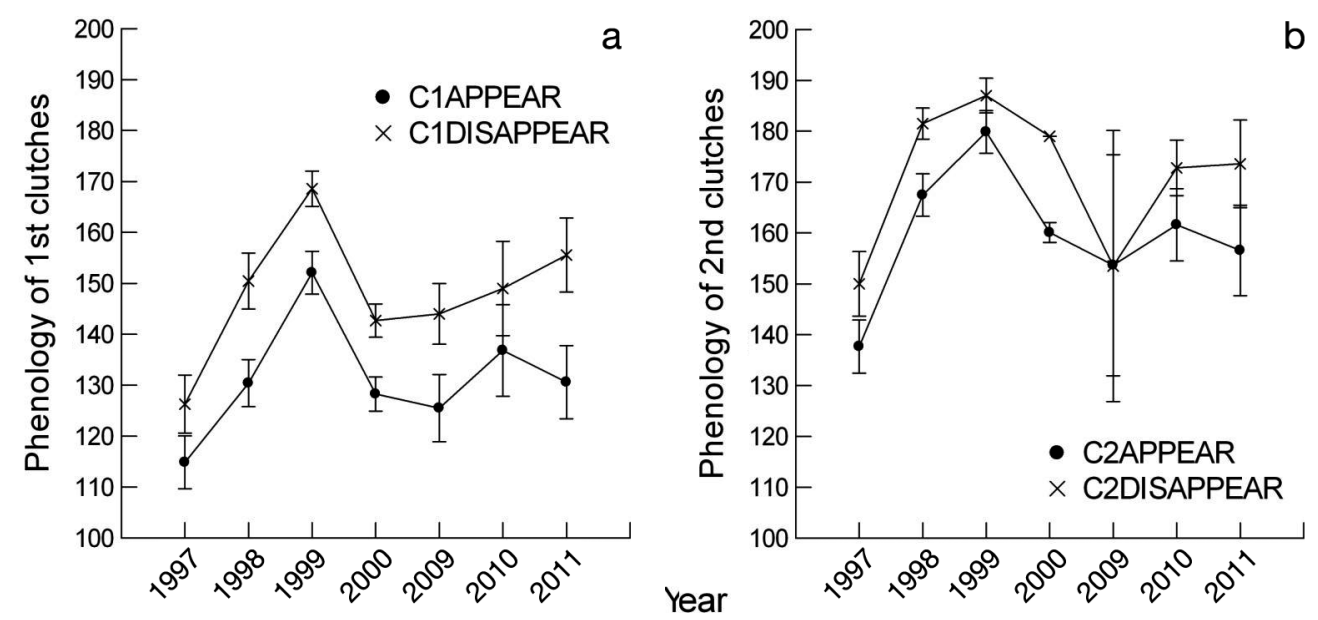

Fig. 3. Gopherus agassizii. Mean number of days from 1 January of each year to the appearance and disappearance of (a) first (C1APPEAR and C1DISAPPEAR) and (b) second clutches (C2APPEAR AND C2DISAPPEAR). Standard errors associated with each phenophase are shown. Convergence of means for second clutches in 2009 was due to small sample size and a missing end date for 1 second clutch 
Table 3. Gopherus agassizii. Tukey's honestly significant difference tests for comparisons among years in the mean number of days from 1 January to phenological events related to clutch production after controlling for maternal effects: days to appearance of first clutch (C1APPEAR), days to disappearance of first clutch (C1DISAPPEAR), days to appearance of second clutch (C2APPEAR), and days to disappearance of second clutch (C2DISAPPEAR). In each case, the mean for the year in the first column is statistically different from the mean or means for the year or years shown in the associated columns to the right. Only significant $(\mathrm{p} \leq 0.05)$ pairwise comparisons are shown

\begin{tabular}{|ccccc|}
\hline \multirow{2}{*}{ Year } & \multicolumn{4}{c|}{$\begin{array}{c}\text { Significant comparisons between years by variable } \\
\text { C2APPEAR }\end{array}$} \\
\cline { 2 - 5 } & C1APPEAR & C1DISAPPEAR & C2DISAPPEAR \\
1997 & 1999 & $1998,1999,2011$ & $1998,1999,2000$ & $1998,1999,2011$ \\
1998 & 1999 & 1999 & - & - \\
1999 & $2000,2009,2011$ & 2000,2009 & 2000,2011 & 2009,2010 \\
2000 & - & - & - & - \\
2009 & - & - & - & - \\
\hline
\end{tabular}

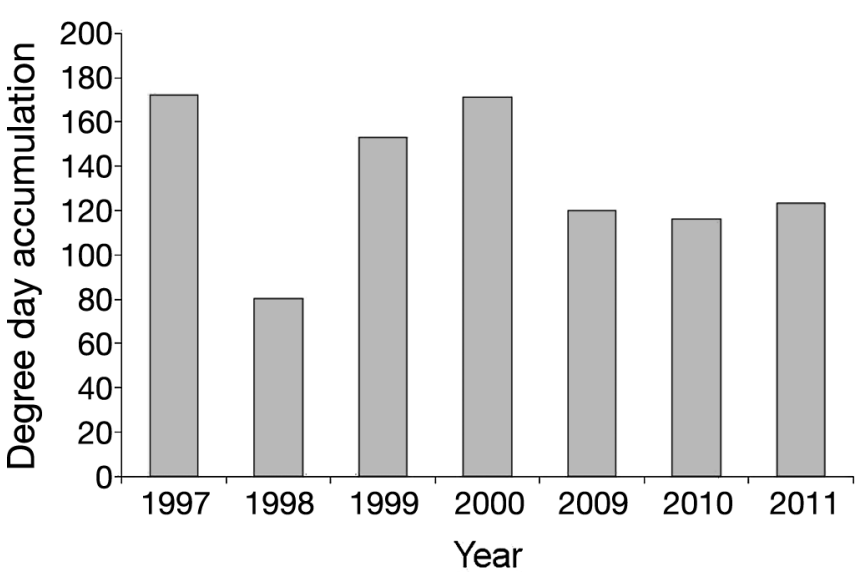

Fig. 4. Gopherus agassizii. Degree day (DD) accumulation at the mean date of appearance of first clutches in desert tortoises for each year of the study. The biofix was set at 1 March at a minimum threshold temperature of $17.77^{\circ} \mathrm{C}$, and DD values were accumulated to the date at which each first clutch was first visible in X-radiographs

\section{DISCUSSION}

The ecology of Agassiz's desert tortoise Gopherus agassizii is greatly influenced by climate, especially temperature and rainfall (Nagy \& Medica 1986). We observed wide variation in clutch phenology at our Mesa study site during 7 field seasons spanning 15 yr. However, most clutch phenophases are correlated with climate, in that phenophases are shorter in warmer years relative to cooler years. It is interesting that DD accumulation to the mean C1APPEAR in 1997 (172 DD) was so different from the same phenophase in 2009 (120 DD). This suggests that the coupling between climate and reproductive physiology is somewhat plastic or that tortoises respond differently under differing climates (Fig. 1). However, the difference in DD accumulation observed is close to the range expected with our X-radiograph interval of up to $10 \mathrm{~d}$. Also puzzling is the failure to accurately predict C1APPEAR in the El Niño year of 1998. It is possible that the method of estimating mean date of first clutch appearance using air temperature works best in years near the 'mean' of climatic variation. In addition, the interaction of rainfall and temperature should be considered in future studies.

This is not the first time such variation has been reported in Gopherus agassizii (sensu lato, Murphy et al. 2011). Comparable data on clutch phenology were presented by Turner et al. (1986) for 2 Mojave Desert populations of $G$. agassizii at Ivanpah Valley and Goffs, both in California. Based on X-radiographs, females at their study sites contained shelled eggs from 21 April to 18 July. As in our study, there was appreciable variation in clutch phenology from year to year. They reported that mean retention time of visible clutches was $22.3 \mathrm{~d}$, with a range of 19 to 25 d. In Washington County, Utah, McLuckie \& Fridell (2002) reported a retention time from initial egg detection to oviposition of $26.6 \mathrm{~d}$. Our mean retention times for various clutches (Table 1) were substantially shorter than those from either of these studies, but our estimates do not include time to oviposition, only visibility in X-radiographs. X-radiograph interval differences between the studies could also affect retention time estimates.

During 2 consecutive years at 2 study sites in California, Wallis et al. (1999) observed no significant difference in the timing of first clutches between years or sites. The discordance of their finding with ours may be related to the longer duration of our study and the likelihood of encompassing greater variation in climate. Variation in mean egg-laying date was also observed in 3 field seasons spanning 8 yr for Gopherus morafkai in Arizona (Averill-Murray et al. 2002). Farther east, in the monsoon-influenced portion of the Sonoran Desert in Arizona, the closely related G. morafkai lays eggs between 6 June and 7 
August (Murray et al. 1996), just before and during the summer rainy season (Van Devender 2002).

As in our study, Wallis et al. (1999) found that the timing of the second clutch was strongly correlated with the timing of the first clutch. In addition, all of our measured phenophases, except for intervals between first and second clutches, differed significantly among years, likely due to cross-correlation of egg production events. The general concordance of phenological differences across time suggests that, while climate appears to cause wide variation in the exact timing and duration of the egg laying season for Gopherus agassizii, egg production time between first and second clutches (inter-clutch interval) is strongly driven by physiological processes that are less sensitive to climatic variation.

Although our analyses suggest that annual variation in climate, particularly temperature (as measured by differential accumulation of DDs during the reproductive season), has an effect on timing of clutch development, other unmeasured variables could be influential. For example, 1 female was the first to produce a visible first clutch in each of 4 consecutive years from 1997 to 2000, suggesting a maternal or genetic effect. Controlling for maternal effects in our analyses still demonstrated significant differences among years. Wallis et al. (1999) listed other sources of unexplained variation in measures of reproductive ecology of Gopherus agassizii, including age, physiological maturity, home range quality, and forage selection. We know that the latter 2 variables differ among females at our study site (Lovich et al. 2011a), but their impact on clutch phenology is, as yet, unquantified.

A major source of unexplained variation for all previous studies of Gopherus agassizii and G. morafkai clutch phenology, including our own, relates to limitations of the methodology. For example, it is impractical to X-radiograph female tortoises daily, as would be required to have precise data on clutch phenology. In fact, federal permits to work with the protected $G$. agassizii now stipulate no more than $3 \mathrm{X}$ radiographs per female per month. In the early years of our study, we were able to X-radiograph females weekly. Variation in X-radiograph frequency undoubtedly contributes to variation in the ability to precisely detect the appearance and disappearance of shelled eggs. This was only exacerbated when tortoise radio signals could not be detected, tortoises could not be removed from deep burrows on days when they were to be X-radiographed, X-radiograph films were unreadable due to problems with equipment, or air temperatures exceeded the tortoise han- dling limit $\left(35^{\circ} \mathrm{C}\right)$ imposed by our federal permit. Use of air temperature as a measure of DD accumulation has its own limitations due to the fact that G. agassizii spends much of its life in burrows buffered from ambient air temperatures. The greater accuracy achieved by Obbard \& Brooks (1987) to predict the date of first nesting in snapping turtles Chelydra serpentina is likely due to their use of more stable water temperatures and an aquatic turtle species.

In spite of the variation observed, we found close association between various clutch phenophases and DD accumulation that allowed us to predict the timing of mean C1APPEAR in subsequent years within 1 to $10 \mathrm{~d}$, if data for 1998 are excluded as an outlier situation. That year was characterized by an El Niño event that had a strong influence on tortoise activity and behavior at our Mesa study site (Ennen et al. 2012a). The high rainfall amount received may have caused tortoises to emerge from hibernation earlier in the year even when temperatures were suboptimal (Medica et. al 1980). This anomaly may explain why mean C1APPEAR occurred at an extremely low DD accumulation value in 1998 (Table 2).

It is interesting to note that 1999, not the El Niño year of 1998, is the year that stands out in Fig. 3 as different from most or all others when comparing mean dates for our phenophases. As noted by Ennen et al. (2012a), tortoise activity and behavior at Mesa in 1999 were comparable to 1998 even though 1999 was the worst drought year (as measured by total winter rainfall and productivity of annual tortoise food plants) out of 3 (1997 to 1999) at Mesa. They concluded that tortoises were able to sequester energy reserves from the El Niño year of 1998 and that allowed them to exhibit comparable levels of activity in a subsequent drought year (1999). The late phenology seen for 1999 in Figs. 2 \& 3, relative to other years, is only partly explained by rainfall in the previous year (Ennen et al. 2012a). The year 1999 was also the second coolest year (after 1998) as shown in Fig. 1. Having 2 cool years in a row may have resulted in the significantly late phenology observed for 1999, again showing the potential importance of climate in a previous year to the reproductive ecology of Gopherus agassizii. Further support for a lag response was shown by Schwanz \& Janzen (2008) and McGaugh et al. (2010) who demonstrated a correlation between previous year DD accumulation to the average onset of nesting for painted turtles Chrysemys picta. In those cases, nesting seasons preceded by warmer winters started earlier than those preceded by cooler winters, supporting our hypothesis for the late phenology observed in 1999. 
The DD accumulation method we used to predict clutch phenology could be tested against data for other sites. If the method proves useful at a broader scale, it could be used to predict changes in clutch phenology based on climate change scenarios that are hypothesized to affect Agassiz's desert tortoise in the vicinity of our studies (Barrows 2011), and perhaps elsewhere. It could also be used as a management tool to minimize disturbance to Gopherus agassizii populations during potentially sensitive phenophases of clutch development. However, wide variability in climate may compromise the effectiveness of our model based on the lack of fit observed in an anomalous year (1998 El Niño).

Given the importance of clutch phenology to timing of emergence from the nest, selection should favor the clutch phenology that results in the highest probability of hatching at a time when conditions are most favorable for neonates. Thus, variation in emergence time from the nest, as associated with clutch phenology, may be related to natural selection responding to differing levels of environmental uncertainty (Gibbons \& Nelson 1978). Producing 2 to 3 clutches a year may ameliorate some of the environmental uncertainty for tortoises by spreading the risk among clutches. Measuring the strength of the relationships between variation in clutch phenology, hatchling growth, and survivorship remain a critical test of the hypothesis advanced by Gibbons \& Nelson (1978). Our research demonstrates that clutch phenology in Gopherus agassizii varies widely with yearto-year fluctuations in climate, but appears to track DD accumulation in a predictable fashion, perhaps to mitigate associated environmental uncertainty. It remains to be determined if tortoises have the ability to respond to future climate change through plasticity in clutch phenology, but our results suggest that this may be possible.

Acknowledgements. Many people assisted us during the course of this study. Field support for this project was provided by interns from the Student Conservation Association, EarthWatch, and volunteers too numerous to list. Special thanks to Ramona Daniels, Rowland Griese, Mark Massar, and Gavin Wright for their long commitment to the support of our project. Al Muth kindly provided accommodations at the Philip L. Boyd Deep Canyon Research Center of the University of California, Riverside, during our research. Mart Westbrooks and Kip Madsen allowed us to use their veterinary facilities to process X-radiographs. Charlie Porter and Annette Burvick of the Flagstaff Medical Center also provided access to processing facilities. Retired US Geological Survey geologist Richard Hereford provided weather data. Research was supported by the California Energy Commission-Public Interest Energy Research (PIER) Program, Cali- fornia Desert District Office of the Bureau of Land Management (BLM), the Palm Springs-South Coast Field Office of BLM, and Joshua Tree National Park. Research was conducted under permits from the US Fish and Wildlife Service, California Department of Fish and Game, and the BLM. We are grateful to the Institutional Animal Care and Use Committee of Northern Arizona University for reviewing and approving our research procedures. Earlier versions of the manuscript benefitted greatly from comments offered by Ken Nussear and Fred Janzen. Any use of trade, product, or firm names is for descriptive purposes only and does not imply endorsement by the US Government.

\section{LITERATURE CITED}

Ashmore GM, Janzen FJ (2003) Phenotypic variation in smooth softshell turtles (Apalone mutica) from eggs incubated in constant versus fluctuating temperatures. Oecologia 134:182-188

Averill-Murray RC, Martin BE, Bailey SJ, Wirt EB (2002) Activity and behavior of the Sonoran desert tortoise in Arizona. In: Van Devender TR (ed) The Sonoran desert tortoise: natural history, biology, and conservation. The University of Arizona Press, Tucson, AZ, p 135-158

Barrows CW (2011) Sensitivity to climate change for two reptiles at the Mojave-Sonoran Desert interface. J Arid Environ 75:629-635

Baskerville GL, Emin P (1969) Rapid estimation of heat accumulation from maximum and minimum temperatures. Ecology 50:514-517

Baxter PC, Wilson DS, Morafka DJ (2008) The effects of nest date and placement of eggs in burrows on sex ratios and potential survival of hatchling desert tortoises, Gopherus agassizii. Chelonian Conserv Biol 7:52-59

Beatley JC (1974) Phenological events and their environmental triggers in Mojave Desert ecosystems. Ecology 55:856-863

Boarman WI, Goodlett T, Goodlett G, Hamilton P (1998) Review of radio transmitter attachment techniques for turtle research and recommendations for improvement. Herpetol Rev 29:26-33

> Bowers JE (2005) El Niño and displays of spring-flowering annuals in the Mojave and Sonoran Deserts. J Torrey Bot Soc 132:38-49

Buhlmann KA, Lynch TK, Gibbons JW, Greene JL (1995) Prolonged egg retention in the turtle Deirochelys reticularia in South Carolina. Herpetologica 51:457-462

Burk JH (1977) Sonoran Desert. In: Barbour MG, Major J (eds) Terrestrial vegetation of California. John Wiley \& Sons, New York, NY, p 869-889

Chhagen A, Stevens PS (2007) Effect of temperature on the development, longevity and oviposition of greenhouse thrips (Heliothrips haemorhoidalis) on lemon fruit. NZ Plant Protection 60:50-55

> Claussen DL, Finkler MS, Smith MM (1997) Thread trailing of turtles: methods for evaluating spatial movements and pathway structure. Can J Zool 75:2120-2128

Ennen JR, Meyer K, Lovich J (2012a) Female Agassiz's desert tortoise activity at a wind energy facility in southern California: the influence of an El Niño event. Nat Sci 4:30-37

Ennen JR, Lovich JE, Meyer K, Bjurlin C, Arundel TR (2012b) Nesting ecology of a desert tortoise (Gopherus agassizii) population at a utility-scale renewable energy facility in southern California. Copeia 2012:222-228 
Ernst CH, Lovich JE (2009) Turtles of the United States and Canada, 2nd edn. Johns Hopkins University Press, Baltimore, DE

Ewert MA, Nelson CE (1991) Sex determination in turtles: diverse patterns and some possible adaptive values. Copeia 1991:50-69

Freedberg S, Ewert MA, Nelson CE (2001) Environmental effects of fitness and consequences for sex allocation in a reptile with environmental sex determination. Evol Ecol Res 3:953-967

Gibbons JW, Nelson DH (1978) The evolutionary significance of delayed emergence from the nest by hatchling turtles. Evolution 32:297-303

Gonzalez-Trapaga R, Aquirre G, Adest GA (2000) Sexsteroids associated with the reproductive cycle in male and female bolson tortoise, Gopherus flavomarginatus. Acta Zool Mex Nueva Ser 80:101-117

Henen BT (2002a) Energy and water balance, diet, and reproduction of female desert tortoises (Gopherus agassizii). Chelonian Conserv Biol 4:319-329

Henen BT (2002b) Reproductive effort and reproductive nutrition of female desert tortoises: essential field methods. Integr Comp Biol 42:43-50

Hinton TG, Fledderman P, Lovich J, Congdon J, Gibbons JW (1997) Radiographic determination of fecundity: Is the technique safe for developing turtle embryos? Chelonian Conserv Biol 2:409-414

> Huey RB, Berrigan D (2001) Temperature, demography, and ectotherm fitness. Am Nat 158:204-210

Iverson JB (1991) Life history and demography of the yellow mud turtle, Kinosternon flavescens. Herpetologica 47: 373-395

> Lewis-Winokur V, Winokur RM (1995) Incubation temperature affects sexual differentiation, incubation time, and posthatching survival in desert tortoises (Gopherus agassizi). Can J Zool 73:2091-2097

Licht P (1983) Seasonal cycles in reptilian reproductive physiology. In: Lamming E (ed) Marshall's physiology of reproduction, Vol 1. Churchill Livingstone, New York, NY, p 206-282

Lovich JE, Daniels R (2000) Environmental characteristics of desert tortoise (Gopherus agassizii) burrow locations in an altered industrial landscape. Chelonian Conserv Biol 3:714-721

Lovich JE, Medica P, Avery H, Meyer K, Bowser G, Brown A (1999) Studies of reproductive output of the desert tortoise at Joshua Tree National Park, the Mojave National Preserve, and comparative sites. Park Science 19:22-24

Lovich JE, Ennen JR, Madrak S, Loughran C, Meyer K, Arundel TV, Bjurlin C (2011a) Long-term post fire effects on spatial ecology and reproductive output of female desert tortoises at a wind energy facility near Palm Springs, California. Fire Ecol 7:75-87

Lovich JE, Ennen JR, Madrak S, Meyer K and others (2011b) Effects of wind energy production on growth, demography and survivorship of a desert tortoise (Gopherus agassizii) population in southern California with comparisons to natural populations. Herpetol Conserv Biol 6:161-174

Luckenbach RA (1982) Ecology and management of the desert tortoise (Gopherus agassizii) in California. In: Bury RB (ed) North American tortoises: conservation and ecology. Wildlife Research Report 12, US Fish and Wildlife Service, Washington, DC

McGaugh SE, Schwanz LE, Bowden RM, Gonzalez JE, Janzen FJ (2010) Inheritance of nesting behavior across natural environmental variation in a turtle with temperature-dependent sex determination. Proc Biol Sci 277: 1219-1226

McLuckie AM, Fridell RA (2002) Reproduction in a desert tortoise (Gopherus agassizii) population on the Beaver Dam Slope, Washington County, Utah. Chelonian Conserv Biol 4:288-294

Medica PA, Bury RB, Luckenbach RA (1980) Drinking and construction of water catchments by the desert tortoise, Gopherus agassizii, in the Mojave Desert. Herpetologica 36:301-304

Mitchell JC (1979) The concept of phenology and its application to the study of amphibian and reptile life histories. Herpetol Rev 10:51-54

Mitchell NJ, Janzen FJ (2010) Temperature-dependent sex determination and contemporary climate change. Sex Dev 4:129-140

Morafka DJ (1994) Neonates: missing links in the life histories of North American tortoises. In: Bury RB, Germano DJ (ed) Biology of North American tortoises. Fish and Wildlife Research 13, United States Department of the Interior, National Biological Survey, Washington, DC, p 161-173

Morafka DJ, Spangenberg EK, Lance VA (2000) Neonatology of reptiles. Herpetol Monogr 14:353-370

Murphy RW, Berry KH, Edwards T, Leviton AE, Lathrop A, Riedle JD (2011) The dazed and confused identity of Agassiz's land tortoise, Gopherus agassizii (Testudines, Testudinidae) with the description of a new species, and its consequences for conservation. ZooKeys 113:39-71

Murray RC, Schwalbe CR, Bailey SJ, Cuneo SP, Hart SD (1996) Reproduction in a population of the desert tortoise, Gopherus agassizii, in the Sonoran Desert. Herpetol Nat Hist 4:83-88

Nagy KA, Medica PA (1986) Physiological ecology of desert tortoises in southern Nevada. Herpetologica 42:73-92

Nussear EK, Esque TC, Haines DF, Tracy CR (2007) Desert tortoise hibernation: temperatures, timing, and environment. Copeia 2007:378-386

Obbard ME, Brooks RJ (1987) Prediction of the onset of the annual nesting season of the common snapping turtle, Chelydra serpentina. Herpetologica 43:324-328

Peterson CC (1996) Ecological energetics of the desert tortoise (Gopherus agassizii): effects of rainfall and drought. Ecology 77:1831-1844

Rautenstrauch KR, Rager AL, Rakestraw DL (1998) Winter behavior of desert tortoises in southcentral Nevada. J Wildl Manag 62:98-104

Rhen T, Lang JW (1995) Phenotypic plasticity for growth in the common snapping turtles: effects of incubation temperature, clutch, and their interaction. Am Nat 146: 726-747

> Rhen T, Lang JW (1999) Incubation temperature and sex affect mass and energy reserves of hatchling snapping turtles, Chelydra serpentina. Oikos 86:311-319

> Rostal DC, Lance VA, Grumbles JS, Alberts AC (1994) Seasonal reproductive cycle of the desert tortoise (Gopherus agassizii) in the eastern Mojave Desert. Herpetol Monogr 8:72-82

> Schwanz LE, Janzen FJ (2008) Climate change and temperature-dependent sex determination: Can individual plasticity in nesting phenology prevent extreme sex ratios? Physiol Biochem Zool 81:826-834

Schwanz LE, Spencer RJ, Bowden RM, Janzen FJ (2010) Climate and predation dominate juvenile and adult recruit- 
ment in a turtle with temperature-dependent sex determination. Ecology 91:3016-3026

Shine R (1999) Why is sex determined by nest temperature in many reptiles? Trends Ecol Evol 14:186-189

Snyder RL (1985) Hand calculating degree days. Agric For Meteorol 35:353-358

Stenseth NC, Mysterud A (2002) Climate, changing phenology, and other life history traits: nonlinearity and matchmismatch to the environment. Proc Natl Acad Sci USA 99:13379-13381

Turner FB, Medica PA, Lyons CL (1984) Reproduction and survival of the desert tortoise (Scaptochelys agassizii) in Ivanpah Valley, California. Copeia 1984:811-820

Turner FB, Hayden P, Burge BL, Roberson JB (1986) Egg production by the desert tortoise (Gopherus agassizii) in California. Herpetologica 42:93-104

US Fish and Wildlife Service (2011) Revised recovery plan for the Mojave population of the desert tortoise (Gopherus agassizii). US Fish and Wildlife Service, Pacific Southwest Region, Sacramento, CA

Valenzuela N (2001) Constant, shift, and natural temperature effects on sex determination in Podocnemis expansa turtles. Ecology 82:3010-3024

Van Devender TR (2002) Natural history of the Sonoran tortoise in Arizona. In: Van Devender TR (ed) The Sonoran desert tortoise: natural history, biology and conservation.

Editorial responsibility: Mike Thompson,

Sydney, Australia
University of Arizona Press, Tucson, AZ, p 3-23

Voigt WG (1972) Thermal ecology of the desert tortoise, Gopherus agassizii, in the Mojave Desert, Kern County, California. Master's thesis, California State University, Hayward, CA

Wallis IR, Henen BT, Nagy KA (1999) Egg size and annual egg production by female desert tortoises (Gopherus agassizii): the importance of food abundance, body size, and date of shelling. J Herpetol 33: 394-408

Walther G-R, Post E, Convey P, Menzel A and others (2002) Ecological responses to recent climate change. Nature 416:389-395

Warner DA, Jorgensen CF, Janzen FJ (2010) Maternal and abiotic effects on egg mortality and hatchling size of turtles: temporal variation in selection over seven years. Funct Ecol 24:857-866

Wilimovsky NJ (1990) Misuses of the term 'Julian Day.'. Trans Am Fish Soc 119:162

> Woodbury AM, Hardy R (1948) Studies of the desert tortoise, Gopherus agassizii. Ecol Monogr 18:145-200

Zimmerman LC, O'Connor MP, Bulova SJ, Spotila JR, Kemp SJ, Salice CJ (1994) Thermal ecology of desert tortoises in the eastern Mojave Desert: seasonal patterns of operative and body temperatures, and microhabitat selection. Herpetol Monogr 8:45-59

Submitted: July 6, 2012; Accepted: September 6, 2012 Proofs received from author(s): November 12, 2012 